\title{
DETERMINATION OF FEMTOSECOND LIFETIMES \\ OF HIGHER EXCITED SINGLET STATES BY MEANS \\ OF TRANSIENT PHOTOPHYSICAL HOLE-BURNING. THE $S_{2}$ STATE OF PHENANTHRENE
}

\author{
Bernhard DICK \\ Max-Planck-Institut für biophysikalische Chemie, Abteilung Laserphysik, Am Fassberg, D-3400 Göttingen, FRG
}

and

Bernhard NICKEL

Max-Planck-Institut für biophysikalische Chemie, Abteilung Spektraskopie, Am Fassberg, D-3400 Göttingen, FRG

Received 26 May 1986

\begin{abstract}
Transient photophysical hole-burning is studied as a method of measuring femtosecond lifetimes of higher excited electronic states of polyatomic molecules. For the 0,0 transition to the $S_{2}$ state of phenanthrene in isopentane/2-methylpentane glass at $4 \mathrm{~K}$ a homogeneous linewidth of $67 \pm 7 \mathrm{~cm}^{-1}$ was found corresponding to a lifetime of $79 \pm 8$ is for this state.
\end{abstract}

\section{Introduction}

Upper electronically excited states of polyatomic molecules are in general very short-lived because of very fast radiationless internal conversion into lower excited states [1]. For aromatic molecules with a singlet ground state, $S_{0}$, estimates of the lifetime $\tau_{n}$ of upper excited singlet states $S_{n}(n>1)$ yielded values typically in the range $10 \mathrm{fs}<\tau_{n}<100 \mathrm{fs}$ [1-6]. Accurate values of $\tau_{n}$ cannot be obtained with conventional methods for reasons discussed below. In the present paper we show that transient photophysical hole-burning (TPPHB) can be used for the determination of lifetimes in the femtosecond range. Before we explain the principles of TPPHB, we briefly expound the different kinds of information obtained from time-domain and frequency-domain experiments and discuss the limitations of lifetime determinations with conventional methods.

Molecules in excited states undergo two kinds of relaxation: population decay and coherence decay. Population decay is the decay of the probability of finding a molecule in an initially pre- pared excited state $|b\rangle$, characterized by a rate constant $\Gamma_{\mathrm{b}}$ whose inverse is commonly called the lifetime of the excited state. Experiments that monitor the population of the excited state, e.g. fluorescence decay measurements, allow to determine this rate constant. Coherence decay is a process characteristic of a pair of levels. When molecules are irradiated by light in resonance with a transition $|a\rangle \rightarrow|b\rangle$, a superposition of these two states is generated resulting in an oscillating dipole moment. The amplitude of this induced dipole moment decays with a rate constant $I_{\mathrm{ab}}$ related to the loss of the initial phase relaxation between the states $|a\rangle$ and $|b\rangle$. The inverse of this relaxation time is, therefore, called phase relaxation time. The lineshape of the transition |a $\rightarrow|b\rangle$ is lorentzian with the homogeneous linewidth twice the rate constant $\Gamma_{\mathrm{ab}}$. The rate constants for population decay and coherence decay are related by:

$\Gamma_{\mathrm{ab}}=\frac{1}{2}\left(\Gamma_{\mathrm{a}}+\Gamma_{\mathrm{b}}\right)+\Gamma^{*}$,

where $\Gamma^{*}$ is the pure-dephasing rate constant. $\Gamma^{*}$ vanishes for isolated molecules in gas phase in the 
absence of collisions, and for molecules in condensed phase at very low temperatures. Under these conditions the coherence-decay rate constant of a transition from the ground state $\left(\Gamma_{\mathrm{a}}=0\right)$ is half the population-decay rate constant,

$\Gamma_{\mathrm{ab}}=\frac{1}{2} \Gamma_{\mathrm{b}}$,

and the homogeneous linewidth of the absorption line can be used to calculate the lifetime. Hence for isolated molecules in gas phase, or molecules in condensed phase at very low temperatures, measurement of the homogeneous width of an absorption line is equivalent to measuring the population decay.

Direct determinations of the lifetimes of higher excited states by measuring fluorescence decay or transient absorption are subject to severe limitations both of technical and principal nature. Such techniques require femtosecond light pulses for excitation and probing. Although such short light pulses have been generated recently [7-9], they are presently available only in a small wavelength interval and require sophisticated equipment. With the up-conversion technique [10] fluorescence decays have been measured with a time resolution down to 1 ps $[11,12]$. However, even with very short light pulses meaningful experiments will not be possible in most cases, because of the difficulty to assign the observed effect to the decay of the initially prepared vibronic state $\mathbf{S}_{n, v}$ (the index $v$ labels the vibrational excitation): the only fluorescence component characteristic of the initially prepared state will be the resonance fluorescence $S_{n, v} \rightarrow S_{0,0}$, i.e. the fluorescence at the wavelength of the excitation pulse. However, since the quantum yield of this fluorescence is very low, Rayleighand Raman-scattered light will be the dominating emission in an interval of $\approx 3000 \mathrm{~cm}^{-1}$ below the excitation wavenumber. Fluorescence at longer wavelengths, however, will contain components originating from relaxed states. A similar dilemma exists for transient-absorption measurements: the absorption spectrum of molecules in a very shortlived vibronic state $S_{n, v}$ will have little structure, and an unambiguous assignment of a transient absorption at a particular wavelength to a transition originating from $\mathrm{S}_{n, v}$ will not be possible.

All previous estimates of lifetimes $\tau_{n}$ of short- lived upper excited singlet states $S_{n}$ were based on indirect methods. In most estimates $[2-6]$ the relation

$\tau_{n}=\tau_{n}^{*} \phi_{\mathrm{f}}^{n \rightarrow 0}$

was used, where $\tau_{n}^{*}$ is the radiative lifetime and $\phi_{\mathrm{f}}^{n \rightarrow 0}$ is the quantum yield of the $S_{n} \rightarrow S_{0}$ fluorescence. $\tau_{n}^{*}$ can be calculated from the strength of the $S_{n} \leftarrow S_{0}$ absorption band [13]. For strong and spectrally isolated $S_{n} \leftarrow S_{0}$ absorption bands an accuracy of $\pm 10 \%$ in the determination of $\tau_{n}^{*}$ can be achieved [13-16]. The measurement of $\phi_{f}^{n \rightarrow 0}$ entails the light-scattering problems mentioned above. With excitation to $S_{n, 0}$ the major part of the $\mathrm{S}_{n} \rightarrow \mathrm{S}_{0}$ fluorescence spectrum is hidden under the much stronger bands of Rayleigh- and Ramanscattered light. With excitation to a vibronic state $S_{n, v}$ some $3000 \mathrm{~cm}^{-1}$ above $S_{n, 0}$ no information on the lifetime of $S_{n, 0}$ is obtained, because internal conversion $S_{n, v} \leadsto S_{m, u}(1 \leqslant m<n)$ is much faster than vibrational relaxation $\mathbf{S}_{n, v} \rightarrow \mathrm{S}_{n, 0}$. Furthermore, again in a spectral range of about 3000 $\mathrm{cm}^{-1}$ the fluorescence $\mathrm{S}_{n, v} \rightarrow \mathrm{S}_{0}$ cannot be measured. The problems resulting from excitation-light scattering are avoided by populating a state $\mathrm{S}_{n}$ through two-photon excitation $S_{n} \leftarrow S_{1} \leftarrow S_{0}$ [5,17-21] or triplet-triplet annihilation (TTA) [6]. However, due to the different selection rules, two-photon excitation will not necessarily populate the state $S_{n}$ observed in the one-photon absorption spectrum. The same restriction holds for TTA; moreover, by TTA different vibronic states $S_{n, v}$ can be populated, and the observed delayed fluorescence from very short-lived states is always the fluorescence from pairs of interacting molecules. In conclusion, if $\tau_{n}$ is very short $(<1 \mathrm{ps}$ ), estimates of $\tau_{n}$ based on eq. (3) yield the order of magnitude of $\tau_{n}$ probably within a factor of two, but no accurate values.

In view of these facts it seems much more reliable to determine the excited-state lifetimes through measurement of homogeneous linewidths. The ideal case of an isolated molecule in a supersonic jet was studied by Amirav et al. [22]. They measured the absorption and fluorescence excitation spectrum of the $S_{2,0} \leftarrow S_{0,0}$ transition in phenanthrene. In both spectra the transition has a lorentzian lineshape with a width of $11 \mathrm{~cm}^{-1}$ 
corresponding to a lifetime of 0.5 ps.

Absorption bands of molecules in condensed phase, especially in glasses or polymer matrices, are inhomogeneously broadened due to slightly different transition frequencies for molecules in different environments (sites). This inhomogeneous broadening ranges from $\approx 1 \mathrm{~cm}^{-1}$ for guest molecules in mixed crystals to more than 100 $\mathrm{cm}^{-1}$ in organic glasses. For long-lived $S_{1}$ states the width of the 0,0 transition in the $S_{1} \leftarrow S_{0}$ absorption spectrum is entirely due to inhomogeneous broadening. If one assumes that inhomogeneous broadening is similar for all electronic transitions of a compound, then the greater width of the absorption lines in transitions to higher excited states can be interpreted in terms of a homogeneous contribution [23]. However, this assumption is difficult to justify.

One method of extracting the homogeneous linewidth from the inhomogeneously broadened system is hole-burning [24]: A narrow-band laser tuned to a wavelength within the inhomogeneously broadened absorption band will excite only molecules which have their transition frequency not more than a few homogeneous linewidths from the laser frequency. As long as these excited molecules have not returned to their electronic ground state, an absorption spectrum of the sample will show a hole at the laser wavelength. Most hole-burning studies [24] have been concerned with the generation and investigation of permanent holes resulting from photochemical reactions. The interest has mainly been in very narrow holes, and the excited states investigated usually have lifetimes in the nanosecond range.

We have chosen a variant of hole-burning which does not require a photochemical reaction, but uses the metastable triplet state in place of a photoproduct [25]. The method should, therefore, be generally applicable. Holes can only be observed within a few triplet lifetimes after excitation. Because of the reversibility of hole formation, many hole-burning experiments at different wavelengths can be performed with the same sample. This is especially important when broad holes are to be burned: with photochemical hole-burning only one experiment could be performed with one sample. The theoretical description of transient hole-burning is similar to the photochemical case. However, since we are interested in transitions with very large homogeneous linewidths, the usual assumption that the inhomogeneous width is dominant cannot be made and a more general discussion of the expected lineshapes is necessary.

For the first series of experiments we chose phenanthrene and fluorene as compounds. The choice of phenanthrene was suggested by the successful supersonic-jet experiment by Amirav et al. [22]. Fluorene was chosen as a reference compound to ensure that the transfer of population to the metastable triplet state indeed was useful for transient hole-burning ${ }^{*}$. The $S_{1} \leftarrow S_{0}$ absorption spectrum of fluorene and the $S_{2} \leftarrow S_{0}$ absorption spectrum of phenanthrene lie in the same wavelength region and have similar oscillator strengths. The triplet lifetimes of fluorene $(5 \mathrm{~s})$ and phenanthrene $(3 \mathrm{~s}$ ) are also similar (table 4.3 of ref. [11]). For the $S_{1} \leftarrow S_{0}$ transition of fluorene, however, narrow holes are expected because of the long lifetime of $S_{1}(10 \mathrm{~ns}$, table 4.3 of ref. [11]).

\section{Theory}

In dilute systems the inhomogeneous broadening of a particular transition can be modelled by a static distribution of transition frequencies: For all molecules the absorption cross section has the same frequency dependence $\sigma\left(\nu-\nu_{0}\right)$, i.e. the same lineshape with the same homogeneous width; the center frequency $\nu_{0}$ is a function of the environment. In this case the inhomogeneous broadening is completely described by the normalized probability distribution function $p\left(\nu_{0}\right)$. In particular, the absorption spectrum $A(\nu)$ of the inhomogeneous ensemble is given by the convolution,

$A(\nu)=N d \int \mathrm{d} \nu_{0} \sigma\left(\nu-\nu_{0}\right) p\left(\nu_{0}\right)$,

where $N$ is the particle density and $d$ the sample thickness.

* When we performed our experiment we were not aware of the fact that transient photophysical hole-burning had already been applied to $S_{1} \leftarrow S_{0}$ absorption bands [25]. 
In eq. (4) the assumption is made that all molecules are in their electronic ground states. When the sample is excited by a laser with frequency $\nu_{e}$ and intensity $I_{e}$, molecules with $\nu_{0}$ close to $\nu_{e}$ will be excited to the high-lying singlet state of interest. Within a few nanoseconds they will decay either back to the ground state or with quantum yield $\phi_{\mathrm{ST}}$ to the lowest triplet state. The triplet state decays with a rate constant $k_{\mathrm{T}}$ to the electronic ground state. Under steady-state conditions the molecules will be in the singlet ground state with probability $n_{\mathrm{S}}$ or in the lowest triplet state with probability $n_{\mathrm{T}}$. On the timescale of our experiments, i.e. the triplet lifetime, the populations of all other states can be neglected, i.e. $n_{\mathrm{S}}+n_{\mathrm{T}}=1$.

The rate equations for this system at a particular position $z$ along the laser light path in the sample are

$$
\begin{aligned}
& \partial n_{\mathrm{S}}\left(\nu_{0} ; z, t\right) / \partial t \\
& =-\sigma\left(\nu_{\mathrm{e}}-\nu_{0}\right) \phi_{\mathrm{ST}} I_{\mathrm{e}}(z, t) n_{\mathrm{S}}\left(\nu_{0} ; z, t\right) \\
& \quad+k_{\mathrm{T}} n_{\mathrm{T}}\left(\nu_{0} ; z, t\right) \\
& \partial I_{\mathrm{e}}(z, t) / \partial z \\
& =-I_{\mathrm{e}}(z, t) N \int \mathrm{d} \nu_{0} p\left(\nu_{0}\right)\left[\sigma\left(\nu_{\mathrm{e}}-\nu_{0}\right)\right. \\
& \left.\quad \times n_{\mathrm{S}}\left(\nu_{0} ; z, t\right)+\sigma_{\mathrm{T}}\left(\nu_{\mathrm{c}}-\nu_{1}\right) n_{\mathrm{T}}\left(\nu_{0} ; z, t\right)\right] .
\end{aligned}
$$

Eq. (6) describes the absorption of the laser light due to the molecules in the ground state, and due to triplet-triplet absorption with cross section $\sigma_{\mathrm{T}}$, originating from the molecules pumped into the triplet state. The attenuation of $I_{\mathrm{c}}$ is in principle non-exponential because $n_{\mathrm{S}}$ and $n_{\mathrm{T}}$ in eq. (6) are functions of $I_{e}$. The steady-state solution of eq. (5) is

$$
n_{\mathrm{S}}\left(\nu_{0} ; z\right)=\left[1+\sigma\left(\nu_{\mathrm{e}}-\nu_{0}\right) \phi_{\mathrm{ST}} I_{\mathrm{e}}(z) / k_{\mathrm{T}}\right]^{-1} .
$$

The absorption of the probe light at frequency $\nu$ is governed by an equation similar to eq. (6),

$$
\begin{aligned}
& \partial I(\nu ; z, t) / \partial z \\
&=-I(\nu ; z, t) N \int \mathrm{d} \nu_{0} p\left(\nu_{0}\right)\left[\sigma\left(\nu-\nu_{0}\right)\right. \\
&\left.\times n_{\mathrm{S}}\left(\nu_{0} ; z, t\right)+\sigma_{\mathrm{T}}\left(\nu-\nu_{1}\right) n_{\mathrm{T}}\left(\nu_{0} ; z, t\right)\right] .
\end{aligned}
$$

Triplet-triplet absorption can in general not be neglected because triplet-triplet absorption cross sections of aromatic molecules in the ultraviolet are usually quite large. However, due to the large number of triplet states in this energy region, the triplet-triplet absorption spectra have usually bandwidths much greater than the homogeneous linewidths of the $S_{n} \leftarrow S_{0}$ absorption spectra of interest. Furthermore, the center frequencies $\nu_{1}$ of the triplet-triplet absorption spectrum are not correlated with the center frequencies $\nu_{0}$ of the singlet-singlet transition under study. That is, an ensemble of molecules with the same value of $\nu_{0}$ will show a whole distribution of values for $\nu_{1}$. If the triplet-triplet absorption spectrum is not known in the spectral range of interest, it is reasonable to assume that the triplet-triplet contribution $A_{\mathrm{TT}}$ to the transient absorption spectrum is constant. Thus eq. (8) yields the steady-state absorption spectrum for constant laser excitation,

$$
A^{*}(\nu)=N d \int \mathrm{d} \nu_{0} p\left(\nu_{0}\right) \sigma\left(\nu-\nu_{0}\right) \bar{n}_{\mathrm{S}}\left(\nu_{0}\right)+A_{\mathrm{TT}},
$$

where $\bar{n}_{\mathrm{S}}$ is the average population of the ground state along the probe light path,

$\bar{n}_{\mathrm{S}}\left(\nu_{0}\right)=d^{-1} \int_{0}^{d} \mathrm{~d} z n_{\mathrm{S}}\left(p_{0} ; z\right)$.

The main obstacle to an analytic solution of eq. (9) is the non-linear coupling of $I_{\mathrm{e}}(z)$ with $n_{\mathrm{S}}\left(\nu_{0} ; z\right)$ through eqs. (6) and (7). However, if the laser intensity is small enough so that only a small fraction of the molecules are pumped into the triplet state, i.e. with

$\sigma\left(\nu_{\mathrm{e}}-\nu_{0}\right) \phi_{\mathrm{ST}} I_{\mathrm{e}}(z) / k_{\mathrm{T}} \ll 1$,

then the expansion of eq. (7) yields

$n_{\mathrm{S}}\left(\nu_{0} ; z\right) \approx 1-\sigma\left(\nu_{\mathrm{e}}-\nu_{0}\right) \phi_{\mathrm{ST}} I_{\mathrm{e}}(z) / k_{\mathrm{T}}$.

This approximation corresponds to the shortburning-time limit in photochemical hole-burning. It brings eq. (6) into the form

$\mathrm{d} I_{\mathrm{e}} / \mathrm{d} z=-a I_{\mathrm{e}}+b I_{\mathrm{e}}^{2}$, 
with

$$
\begin{aligned}
a= & A\left(\nu_{\mathrm{e}}\right) / d, \\
b= & N \phi_{\mathrm{ST}} k_{\mathrm{T}}^{-1} \int \mathrm{d} \nu_{0} p\left(\nu_{0}^{\prime}\right) \sigma\left(\nu_{\mathrm{e}}-\nu_{0}\right) \\
& \times\left[\sigma\left(\nu_{\mathrm{e}}-\nu_{0}\right)-\sigma_{T}\left(\nu_{\mathrm{e}}-\nu_{1}\right)\right] .
\end{aligned}
$$

The solution of eq. (13) is

$$
I_{\mathrm{e}}(z)=a I_{0}\left[b I_{0}+(a-b) I_{0} \exp (a z)\right]^{-1} \text {. }
$$

With eq. (12) the average population $\bar{n}_{\mathrm{S}}\left(\nu_{0}\right)$ becomes a linear function of the average laser intensity, $\bar{I}_{\mathrm{e}}$, defined in the same way as $\bar{n}_{\mathrm{S}}$ in eq. (10),

$\bar{n}_{\mathrm{S}}\left(\nu_{0}\right)=1-\sigma\left(\nu_{\mathrm{e}}-\nu_{0}\right) \phi_{\mathrm{ST}} \bar{I}_{\mathrm{e}} / k_{\mathrm{T}}$.

We would like to note at this point that it was not necessary to employ the thin-sample approximation to obtain this result. Hence the optical density of the sample is not a critical parameter in our experiments.

The hole-shape function $H(\nu)$ is defined as the frequency-dependent part of the difference of the absorption spectra with and without laser excitation,

$H(\nu)=A(\nu)-A^{*}(\nu)+A_{\mathrm{TT}}$.

Eqs. (4), (9), and (16) yield

$$
\begin{aligned}
H(\nu)= & N d \phi_{\mathrm{ST}} \bar{I}_{\mathrm{e}} k_{\mathrm{T}}^{-1} \\
& \times \int \mathrm{d} \nu_{0} p\left(\nu_{0}\right) \sigma\left(\nu-\nu_{0}\right) \sigma\left(\nu_{\mathrm{e}}-\nu_{0}\right) .
\end{aligned}
$$

So far we have assumed a steady state. A more rigorous time-dependent treatment of the problem leads to the same result when eq. (11) is fulfilled for the time-averaged laser intensity. In addition the laser peak intensity must obey the relation

$\sigma\left(\nu_{\mathrm{e}}-\nu_{0}\right) I_{\text {peak }} \tau_{n} \ll 1$,

where $\tau_{n}$ is the lifetime of the initially prepared excited singlet state $S_{n}$. In our experiments on phenanthrene the second condition is easily fulfilled since $\tau_{n}<1 \mathrm{ps}$. Condition (11) is fulfilled when the depth of the hole is proportional to the average laser intensity, which may be checked experimentally.

For a quantitative lineshape analysis reasona- ble assumptions have to be made for $\sigma\left(\nu-\nu_{0}\right)$ and $p\left(\nu_{0}\right)$. For the zero-phonon line, the natural choice for the homogeneous lineshape function $\sigma\left(\nu-\nu_{0}\right)$ is a lorentzian $\sigma_{L}$ corresponding to an exponentially decaying state,

$\sigma_{\mathrm{L}}\left(\nu-\nu_{0}\right)=\left(a_{0} \Gamma / \pi\right)\left[\left(\nu-\nu_{0}\right)^{2}+\Gamma^{2}\right]^{-1}$,

where $a_{0}$ is the integral of $\sigma_{L}\left(\nu-\nu_{0}\right)$ over the frequency $\nu$. This lineshape has a full width at half maximum (fwhm) of $B_{\mathrm{L}}=2 \Gamma$. The corresponding lifetime $\tau$ is given by

$\tau=\left(2 \pi c B_{L}\right)^{-1}$,

where $B_{\mathrm{L}}$ is measured in wavenumber units and $c$ is the speed of light. In condensed phase, apart from the zero-phonon line, in principle always a phonon sideband has to be considered [24]. It results from simultaneous excitation of the guestmolecule transition of interest and low-frequency lattice modes of the host. The lifetime $\tau_{n}$ is only available from the lorentzian zero-phonon line. Therefore, for the present purpose, hole-burning is only applicable in cases where the zero-phonon lines make an appreciable contribution to the absorption spectrum. In the case of phenanthrene in alkane glass, studied here, a contribution of phonon sidebands to the hole spectrum or the absorption spectrum was not detectable.

If the distribution of center frequencies $\nu_{0}$ is the result of many independent perturbations due to different local environment, fields, and strain, then the best choice for the inhomogeneous distribution function $p\left(\nu_{0}\right)$ is a gaussian,

$$
\begin{aligned}
& p\left(\nu_{0}\right)=(\gamma \sqrt{2 \pi})^{-1} \exp \left[-\left(\nu_{0}-\nu_{\mathrm{A}}\right)^{2} / 2 \gamma^{2}\right], \\
& B_{\mathrm{G}}=(8 \ln 2)^{1 / 2} \gamma,
\end{aligned}
$$

where $\nu_{A}$ is the average zero-phonon center frequency of the inhomogeneous ensemble, and $B_{\mathrm{G}}$ is again the full width at half maximum. The absorption band in the absence of laser excitation can now be calculated according to eq. (4),

$$
\begin{aligned}
A(\nu)= & N d(\gamma \sqrt{2 \pi})^{-1} a_{0} \\
& \times \operatorname{Re}\left[w\left(\left(\nu-\nu_{\mathrm{A}}+\mathrm{i} \Gamma\right) / \gamma \sqrt{2}\right)\right],
\end{aligned}
$$

where $w(z)$ is the complex error function [26]. In 
the case of dominant inhomogeneous broadening $\left(B_{\mathrm{G}}>B_{\mathrm{L}}\right.$ ) the central part of the absorption band is very nearly gaussian.

For the hole-shape function $H(v)$ the result of the triple convolution of eq. (18) is

$$
\begin{aligned}
& H(\nu)= N d \phi_{\mathrm{ST}} \bar{I}_{\mathrm{e}} k_{\mathrm{T}}^{-1} a_{0}^{2}\left[\sqrt{\pi / 2} / \gamma \Gamma\left(\nu_{\mathrm{e}}-\nu\right)\right] \\
& \times \operatorname{Re}\left[w\left(q_{1}\right) /\left(\nu_{\mathrm{e}}-\nu+2 \mathrm{i} \Gamma\right)\right. \\
&\left.+w\left(q_{2}\right) /\left(\nu_{\mathrm{e}}-\nu-2 \mathrm{i} \Gamma\right)\right] \\
& q_{1}=\left(\nu_{\mathrm{e}}-\nu_{\mathrm{A}}+\mathrm{i} \Gamma\right) / \gamma \sqrt{2} \\
& q_{2}=\left(\nu-\nu_{\mathrm{A}}+\mathrm{i} \Gamma\right) / \gamma \sqrt{2}
\end{aligned}
$$

$H(v)$ reduces to simple expressions in the limits $B_{\mathrm{G}} \ll B_{\mathrm{L}}$ and $B_{\mathrm{G}} \gg B_{\mathrm{L}}$ corresponding to the purely homogeneous and purely inhomogeneous case, respectively.

In the homogeneous case, $B_{\mathrm{G}}=0$, the distribution function $p\left(\nu_{0}\right)$ reduces to the $\delta$-function, and the hole shape as well as the shape of the absorption band are given by the homogeneous lineshape function $\sigma_{L}\left(\nu-\nu_{A}\right)$ centered at $\nu_{A}$, independent of the laser frequency $\nu_{\mathrm{e}}$. The widths of the hole and of the absorption line are equal to the width of the lorentzian, $B_{\mathrm{L}}$, and the whole absorption band is bleached.

In the inhomogeneous limit $B_{\mathrm{G}} \gg B_{\mathrm{L}}$ the hole-shape function reduces to

$H(\nu) \propto\left[\left(\nu_{\mathrm{e}}-\nu\right)^{2}+4 \Gamma^{2}\right]^{-1}$.

This hole-shape function is again lorentzian, but centered at the frequency of the laser, $\nu_{\mathrm{e}}$, and with twice the width of the homogeneous line: $B_{\mathrm{H}}=$ $2 B_{\mathrm{L}}$.

Numerical simulations based on eq. (24) show that in the intermediate case, when $B_{\mathrm{L}}$ and $B_{\mathrm{G}}$ are of similar magnitude, the width of the hole, $B_{\mathrm{H}}$, lies in the interval $B_{\mathrm{L}}<B_{\mathrm{H}}<2 B_{\mathrm{L}}$. The hole shape will be symmetric only if the laser is tuned to the center frequency of the inhomogeneous distribution, i.e. $\nu_{\mathrm{e}}=\nu_{\mathrm{A}}$. In this case, however, the hole shape is very similar to a lorentzian. An example is shown in fig. 1 . Three hole shapes are simulated for the same homogeneous width $\left(B_{\mathrm{L}}=\right.$ 1). Curves a and $c$ display the inhomogeneous and homogeneous limits, whereas curve $b$ corresponds

\section{Holeshapes}

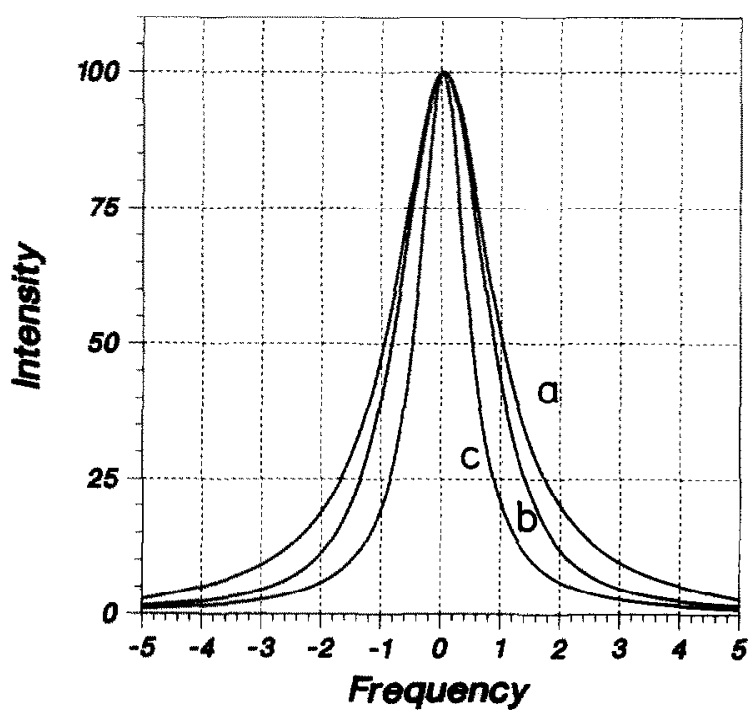

Fig. 1. Simulated hole-shapes. The width $B_{L}$ of the homogeneous line is one frequency unit for all curves. (a) shows the inhomogeneous limit $\left(B_{\mathrm{G}} \rightarrow B_{\mathrm{L}}\right)$, (c) the homogeneous limit

$\left(B_{\mathrm{G}} \ll B_{\mathrm{L}}\right.$ ), and (b) an intermediate case with $B_{\mathrm{G}}=3 B_{\mathrm{L}}$.

to $B_{\mathrm{G}}=3 B_{\mathrm{L}}$. Apparently the central part of the absorption band contains the gaussian information, whereas the central part of the hole carries the lorentzian information of the transition.

When $B_{\mathrm{L}}$ and $B_{\mathrm{G}}$ are of similar size, they can be calculated through simultaneous deconvolution of the absorption and hole lineshapes. To perform this deconvolution, we calculated the widths of the absorption band, $B_{A}$, and of the hole, $B_{H}$, for a large range of the ratio $B_{\mathrm{L}} / B_{\mathrm{G}}$. In fig. 2 the resulting ratios $x=\mathrm{B}_{\mathrm{L}} / \mathrm{B}_{\mathrm{H}}$ and $y=\mathrm{B}_{\mathrm{G}} / \mathrm{B}_{\mathrm{A}}$ are plotted against the ratio $B_{\mathrm{H}} / B_{\mathrm{A}}$. The latter is directly available from the experiment, $B_{\mathrm{H}} / B_{\mathrm{A}}=0$. corresponding to the inhomogeneous limit, and $B_{\mathrm{H}} / B_{\mathrm{A}}=1$ corresponding to the homogeneous limit. The values of $x$ and $y$ belonging to $a$ particular ratio $B_{H} / B_{A}$ yield the desired values for $B_{L}$ and $B_{G}$ through

$B_{\mathrm{L}}=x B_{\mathrm{H}}, \quad B_{\mathrm{G}}=y B_{\mathrm{A}}$. 


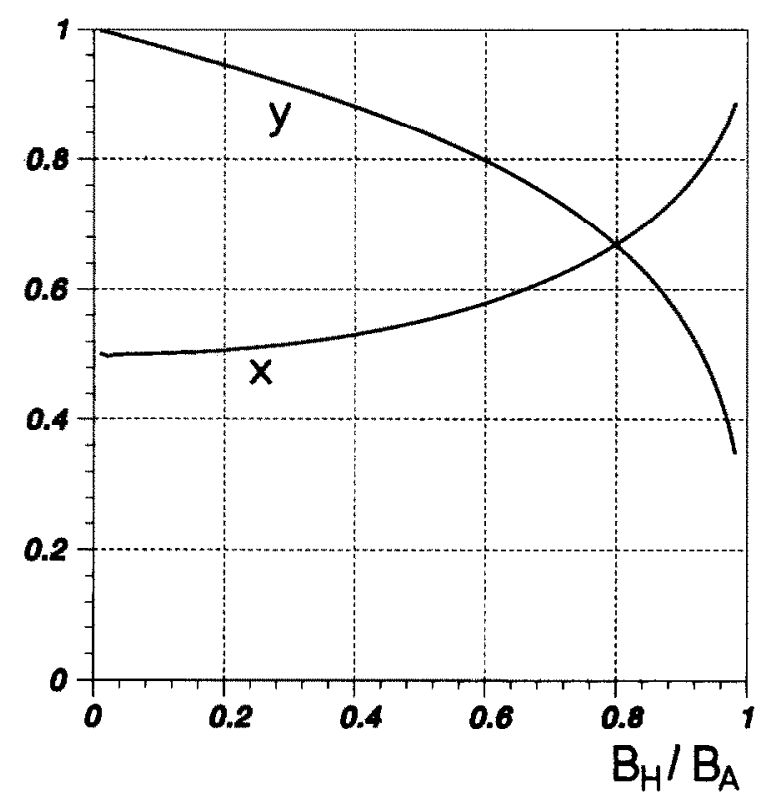

Fig. 2. Deconvolution factors $x=B_{\mathrm{L}} / B_{\mathrm{H}}$ and $y=B_{\mathrm{G}} / B_{\mathrm{A}}$ for calculating the homogeneous width $B_{\mathrm{L}}$ and the inhomogeneous width $B_{\mathrm{G}}$ from the ratio $B_{\mathrm{H}} / B_{\mathrm{A}}$ of the width of the hole to that of the absorption band. A gaussian inhomogeneous distribution and hole-burning into the center of the absorption band are assumed. For discussion see text.

\section{Experimental}

\subsection{Substances}

Zone-refined phenanthrene and fluorene were used. The phenanthrene was free of its common impurities dibenzothiophene and anthracene. Isopentane and 2-methylpentane were rectified over a $1 \mathrm{~m}$ column. Before use both solvents were additionally purified and dried by column chromatography with basic aluminum oxide and stored in glass vessels closed with grease-free PTFE valves.

\subsection{Samples}

The most serious experimental problem was the preparation of samples forming a clear glass at 4 K. Normal high-quality fluorescence or absorption cuvettes cracked invariably at low temperatures. Home-made absorption cuvettes (made of
Suprasil fused silica tube of $12 \times 12 \mathrm{~mm}$ outer cross section) turned out to be mechanically sufficiently stable. Two opposite faces of these cuvettes were polished at the outside. In thoroughly degassed samples the glass very often formed cracks when the temperature was decreased below $40 \mathrm{~K}$. We found that formation of cracks could be largely suppressed through an inert gas inside the cell, preferably helium. Probably solvent evaporation and solute crystallization at the surface of the liquid are responsible for the formation of cracks.

Samples were prepared in three steps. Firstly, the suitable amount of a stock solution of the aromatic hydrocarbon in hexane was filled into the cuvette and the solvent was evaporated in vacuo. Secondly, for preparing a mixed solvent of any desired composition, the single components were first distilled in vacuo from storage vessels into a thin graduated and calibrated tube and from that tube into the cuvette (cooled with dry ice to $195 \mathrm{~K}$ ). Finally, the cuvette was cooled to 77 $\mathrm{K}$, filled with 0.3 bar of helium, and sealed off. The volume of the solution in the cuvette was about $1 \mathrm{~cm}^{3}$ at room temperature.

\subsection{Experimental arrangement}

The experimental arrangement was essentially a luminescence spectrometer built for measuring short-lived phosphorescence or delayed fluorescence with pulsed laser excitation (see fig. 3 ). The cuvette was placed in a continuous-flow cryostat (Oxford Instruments CF 204). The sample holder had two apertures of $2 \mathrm{~mm}$ diameter, just above the bottom of the cuvette and close to its windows. The optical axis of the cryostat coincided with the optical axis of a fast mechanical chopper (two disks of $200 \mathrm{~mm}$ diameter on a common shaft, vacuum-tight housing with two pairs of slits, reduced pressure of $\approx 10 \mathrm{mbar}$, up to $600 \mathrm{rps}$ ). The laser used for hole-burning was a frequency-doubled dye laser (Lambda Physik FL 2000), pumped by an excimer laser (Lambda Physik EMG 50) and synchronized to the chopper; the number $n$ of chopper rotations per laser pulse could be varied according to the average excitation power desired. The light source for measuring the absorption spectrum was a xenon arc lamp. Predominant 


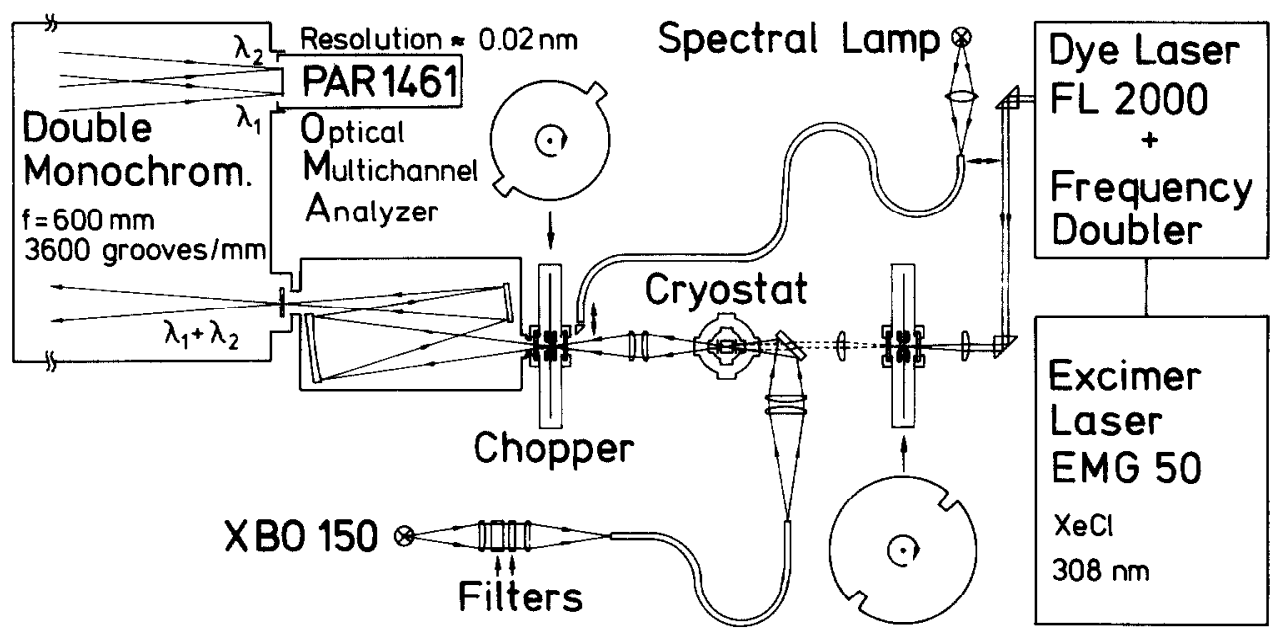

Fig. 3. Schematic representation of the experimental arrangement.

vertical polarization of the measuring light was effected by reflection from a thick silica plate. The frequency-doubled laser light was also vertically polarized. The light transmitted by the sample was dispersed with a home-made double monochromator with $600 \mathrm{~mm}$ focal length. With two holographic gratings having 3600 grooves $/ \mathrm{mm}$ the reciprocal linear dispersion was $0.2 \mathrm{~nm} / \mathrm{mm}$ in first order and the maximum resolution was $0.01 \mathrm{~nm}$. The monochromator has two selectable exits, one of which was supplied with a slit and a photomultiplier (not shown in fig. 3) for measuring the absorption spectrum of the sample over a wide range. The second exit was connected with an optical multichannel analyzer (OMA) (Princeton Applied Research, OMA-III system with imageintensifier camera). The useful spectral range of the OMA at $300 \mathrm{~nm}$ was $3.5 \mathrm{~nm}$ imaged onto 700 intensified channels, and the practical resolution was $0.02 \mathrm{~nm}$ as tested by the resolution of the mercury doublet at $313.155 / 313.183 \mathrm{~nm}$. For measuring the laser wavelength or for spectral calibration of the OMA, the light of the laser or of a spectral lamp was brought to the luminescence slit of the chopper via an UV fiber light guide.

Due to the long triplet lifetime of phenanthrene ( $3 \mathrm{~s})$ even with repetition rates down to $2 \mathrm{~Hz}$ (the lowest repetition rate used in our experiments) the measurement was essentially a steady-state mea- surement. Each spectrum was taken in $30 \mathrm{~s}$, numerically accumulated as 100 scans of $300 \mathrm{~ms}$ exposure time. Each hole-burning experiment consisted of measuring four spectra: one with the laser switched off, one $15 \mathrm{~s}$ after the laser was turned on, one $40 \mathrm{~s}$ after the laser was switched off again, and one with all light sources blocked to obtain the diode background of the camera. After subtraction of this background, the difference of the decadic logarithms of the first two spectra gave the hole spectrum. The third spectrum served as a test to detect any photochemical degradation of the sample or a change in experimental parameters (such as lamp-current fluctuations etc.). For applications of the method to compounds with short-lived triplet states the image-intensifier camera can be gated and a pulsed xenon lamp can be used.

\section{Results and discussion}

In fig. 4a the $S_{2} \leftarrow S_{0}$ absorption spectrum of phenanthrene and in fig. $4 \mathrm{~b}$ the $S_{1} \leftarrow S_{0}$ absorption spectrum of fluorene in 2-methylpentane/ isopentane glass at $4 \mathrm{~K}$ are shown. In the wavelength region $270-310 \mathrm{~nm}$ the absorption spectra of both compounds display distinct origin bands and vibronic transitions with similar widths. The 


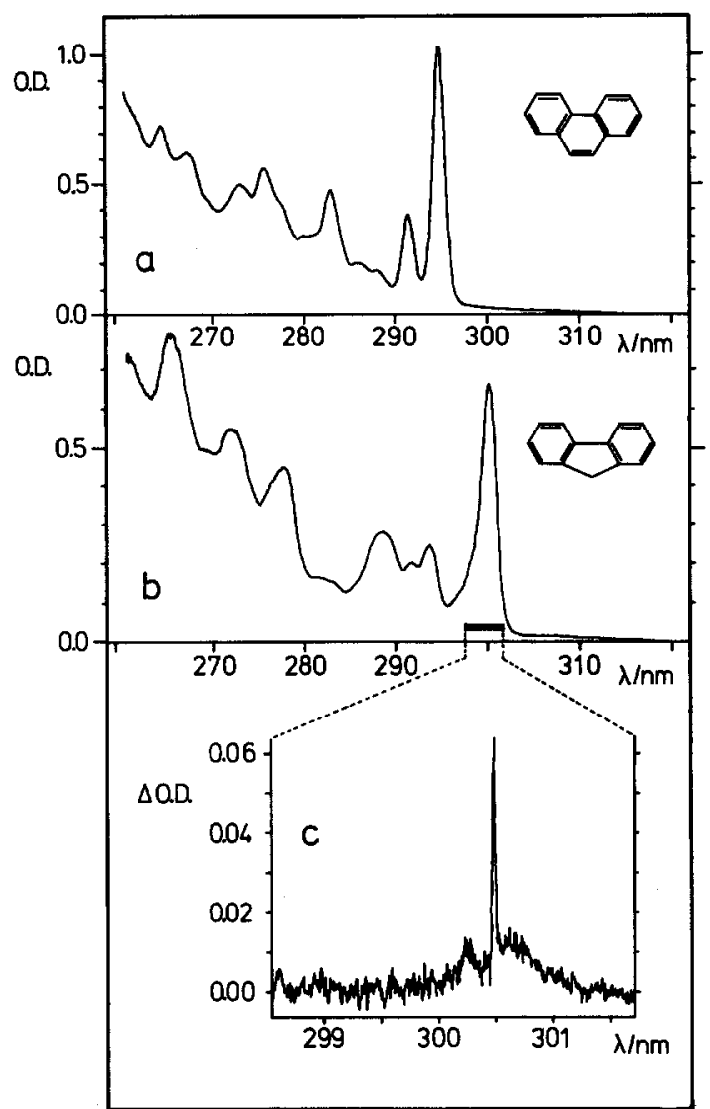

Fig. 4. Absorption spectra of phenanthrene (a) and fluorene (b) in isopentane $/ 2$-methylpentane glass at $4 \mathrm{~K}$. The spectra refer to air as reference. The optical density (OD) is defined as usual: $\mathrm{OD}=\log _{10}\left(I_{0} / I\right)$. The length of the horizontal bar corresponds to the spectral region seen by the OMA camera. (c) Transient hole in the 0,0 transition of the $S_{1} \leftarrow S_{0}$ absorption band of fluorene. The width of the zero-phonon line shows the resolution of our apparatus.

important difference is that the $S_{1}$ state of fluorene has a lifetime of $10 \mathrm{~ns}$, whereas the $S_{2}$ state of phenanthrene has a lifetime of less than 0.5 ps. Therefore, completely different hole-burning spectra are expected for both compounds in spite of the strong similarity of the absorption spectra.

The hole obtained through laser excitation in the 0,0 transition of the $S_{1} \leftarrow S_{0}$ absorption band of fluorene is shown in fig. 4c. It displays a sharp zero-phonon line of $1.5 \mathrm{~cm}^{-1}$ width (3.5 OMA channels). This width is determined by the resolution of our apparatus, the limiting factors being the linear dispersion of the monochromator $(0.5$ $\mathrm{cm}^{-1}$ per OMA channel at $300 \mathrm{~nm}$ ) and the diode cross talk in the OMA camera $(\approx 30 \%$ between adjacent channels). This result demonstrates that transfer of population to the metastable triplet state indeed can be used for transient hole-burning. With the present apparatus we can expect to make reliable measurements of hole widths down to $\approx 5 \mathrm{~cm}^{-1}$, corresponding to lifetimes up to $\approx 2$ ps.

Transient hole-burning can be used for the determination of accurate vibrational frequencies of molecules in excited states through the observation of non-resonant holes. Since the hole-burning process removes molecules from the electronic ground state, the missing molecules will not only leave a hole in the transition which is burned (resonant hole), but also in all other transitions (non-resonant holes) [24]. If the inhomogeneous distributions for the burned and the probed transitions are strongly correlated, the non-resonant holes will be also narrow. This correlation is usually very good for vibronic transitions belonging to the same electronic transition: perfect correlation means that all molecules have the same vibrational frequencies in the excited state, and that the inhomogeneous broadening is entirely due to shifts of the electronic origin for molecules at different sites.

We observed narrow non-resonant holes in the absorption spectrum of fluorene with the laser tuned to several different vibronic transitions. The absorption spectra were taken in overlapping sections through changing the center wavelength of the monochromator in steps of $2 \mathrm{~nm}$. Fig. 5 shows four such sections with the laser tuned to the 0,0 transition of the $S_{1} \leftarrow S_{0}$ absorption band. The holes were burned with a laser intensity higher than for the spectrum in fig. 4 resulting in the appearance of broad phonon-sideband holes. The three prominent non-resonant holes observed in this wavelength region are labelled with their wavenumber shift relative to the resonant hole in the 0,0 transition.

For hole-burning experiments with phenanthrene we chose the 0,0 transition $S_{2,0} \leftarrow S_{0,0}$ and the first vibronic transition $S_{2,1} \leftarrow S_{0,0} 400 \mathrm{~cm}^{-1}$ above the origin. Both bands are well separated from other bands and show highly symmetric band 


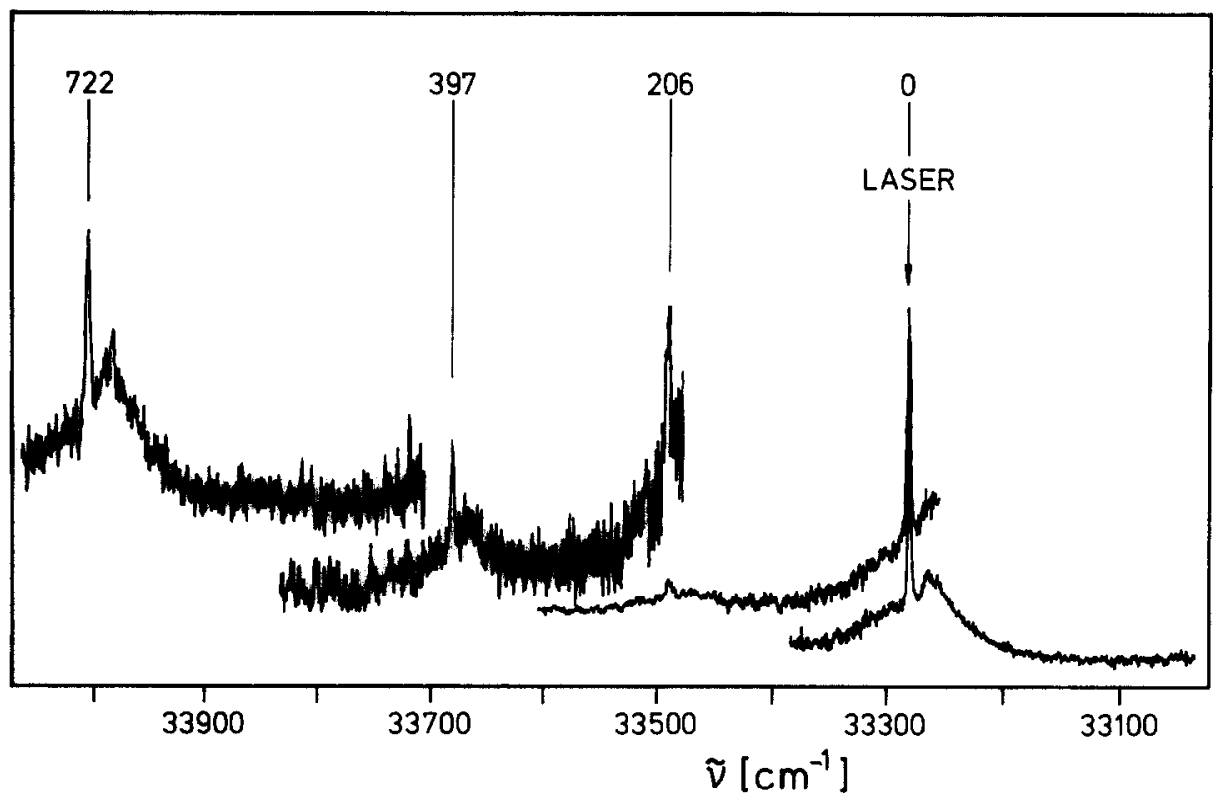

Fig. 5. Non-resonant satellite holes in the fluorene $S_{1} \leftarrow S_{0}$ absorption band. The laser was tuned to the 0,0 transition. Absorption spectra were taken in overlapping sections through decreasing the center wavelength of the monochromator in steps of 2 nm. The holes are labelled by their vibrational wavenumbers.

shapes, indicating that both bands belong to single vibronic levels of phenanthrene. The hole obtained with the laser tuned to the center of the 0,0 transition is shown in fig. $6 \mathrm{~b}$. Fig. 6a shows the absorption spectrum taken under the same conditions just before laser irradiation.

The fwhm of the hole, $110 \mathrm{~cm}^{-1}$, is much greater than in the case of fluorene. Nevertheless, it is clearly narrower than the absorption band with a fwhm of $163 \mathrm{~cm}^{-1}$. In addition, when the laser wavelength is varied, the center of the hole also shifts in the same direction as the laser wavelength, as expected for hole-burning. However, when the hole is not burned near the center of the absorption line, the hole shape is unsymmetric and the peak is not exactly at the laser wavelength. This is a consequence of the fact that the homogeneous width is not much smaller than the inhomogeneous width. Hence the simple formula (25) for the inhomogeneous limit, which is commonly used in hole-burning studies, no longer applies, and the general formula (24) must be used instead.

The analysis of the band shapes is easiest when

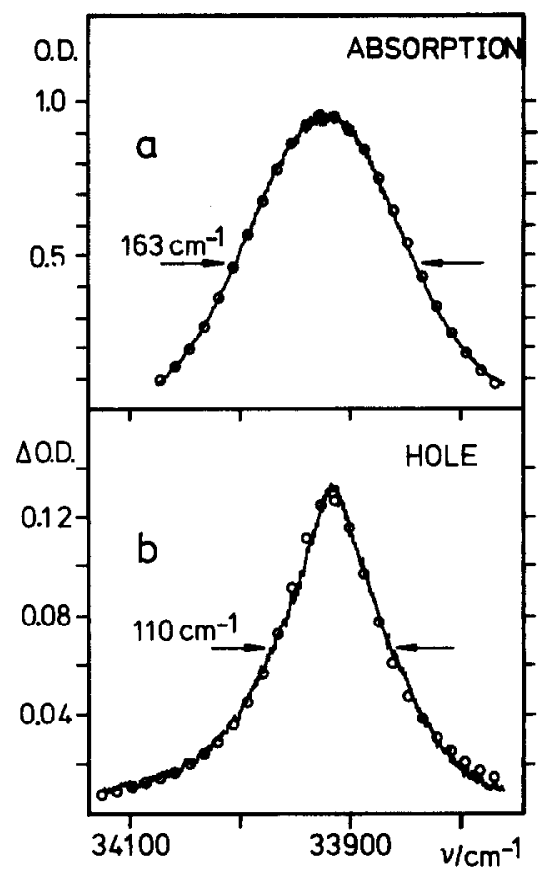

Fig. 6. Hole-burning in the 0,0 transition of the $S_{2} \leftarrow S_{0}$ absorption band of phenanthrene. (a) Inhomogeneously broadened absorption line; circles indicate gaussian fit. (b) Hole obtained with excitation near the center of the absorption line; circles indicate lorentzian fit. 
the hole shape is symmetric due to burning into the center of the absorption band. This is the case in fig. 6. Here, a gaussian is fitted to the absorption band and a lorentzian to the hole, both indicated by open circles. The fit yielded a width of $162.9 \pm 1.0 \mathrm{~cm}^{-1}( \pm 0.6 \%)$ for the gaussian and of $110.3 \pm 3.4 \mathrm{~cm}^{-1}( \pm 3.1 \%)$ for the lorentzian. This is in very good agreement with the statement made in section 2 that, even in the case of similar homogeneous and inhomogeneous widths (vide infra), the absorption band is almost gaussian, whereas the hole is almost lorentzian. In addition, the near-lorentzian shape of the hole proves the dominance of the zero-phonon line in the $S_{2,0} \leftarrow$ $S_{0,0}$ absorption: If burning would be predominantly into a phonon sideband, the hole would be gaussian. The depth of the hole is 0.13 units of optical density, corresponding to the transfer of $\approx 10 \%$ of the phenanthrene molecules into the triplet state. The baseline of the fitted lorentzian is 0.004 optical density units above the measured baseline. This is not significantly different from zero within our experimental accuracy. Hence the contribution from triplet-triplet absorption is very small. (The triplet-triplet absorption spectrum is not known in this spectral range.)

We can now apply the simple deconvolution technique outlined in section 2 . The ratio $B_{\mathrm{H}} / B_{\mathrm{A}}$ is 0.675 , leading to $x=0.606$ and $y=0.758$ (cf. fig. 2). These yield $B_{\mathrm{G}}=123.5 \mathrm{~cm}^{-1}$ and $B_{\mathrm{L}}=66.9$ $\mathrm{cm}^{-1}$ from eq. (26). The unicertainty in these values should be the sum of the relative uncertainties in the fits for $B_{A}$ and $B_{H}$, i.e. $\pm 3.7 \%$, plus a contribution from the resolution of our apparatus. We believe that $\pm 10 \%$ is a conservative estimate. With eq. (21) we calculate a lifetime for the $S_{2,0}$ state of $79 \pm 8 \mathrm{fs}$. This lifetime must be compared to the lifetime of 500 fs found for the same transition in the isolated molecule in a supersonic jet [22]. In order to interpret the homogeneous linewidth entirely in terms of a population lifetime, pure dephasing must be excluded as a possible source of line broadening. Phenanthrene and fluorene have similar size and shape, and they very likely occupy similar sites in the organic glass. Hence both compounds should experience interactions with the host of similar type and strength. Pure dephasing processes are tempera- ture induced, and their onset should be observed as an increase in the linewidth when the temperature is raised. No significant broadening of the hole was observed for phenanthrene when the temperature was raised to $15 \mathrm{~K}$. At this temperature the narrow hole observed in the $S_{1,0} \leftarrow S_{0,0}$ absorption band of fluorene had a width of less than $4 \mathrm{~cm}^{-1}$. Hence we believe that pure dephasing is unimportant in the $S_{2}$ state at $4 \mathrm{~K}$.

To investigate the effect of vibrational excess energy on the radiationless decay rates we included the vibronic $S_{2,1}$ state $\approx 400 \mathrm{~cm}^{-1}$ above the origin into our hole-burning study. The inclusion of a vibronic state also allowed the observation of non-resonant holes. Fig. 7 shows the holes observed in the transition to $S_{2,0}$ (right-hand side) and the first vibronic state $S_{2,1}$ (left-hand side). The laser was tuned to the center of the 0,0 transition in the upper part and to the center of the vibronic band in the lower part. Thus curves $b$ and $c$ are resonant holes whereas curves $a$ and $d$

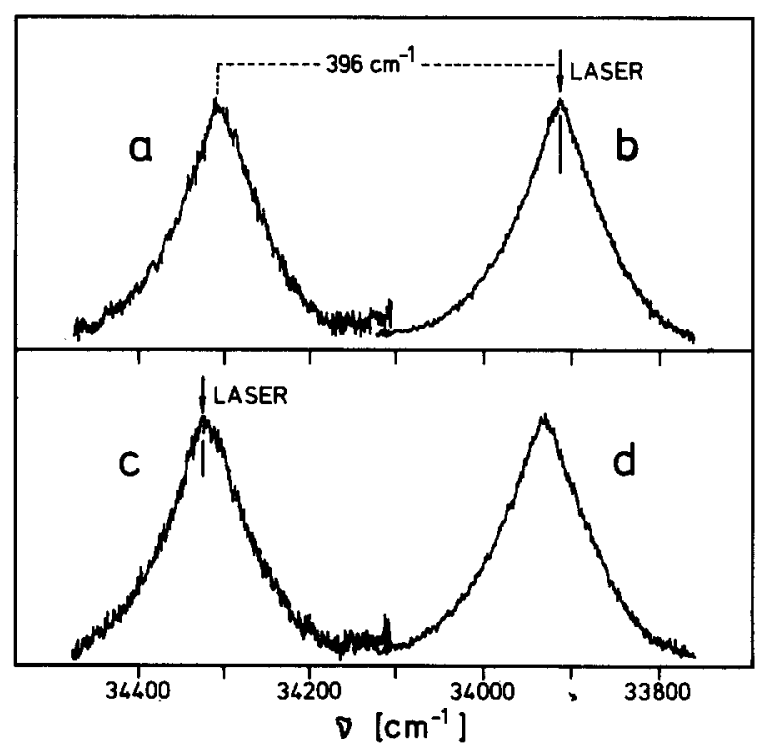

Fig. 7. Resonant and non-resonant holes in the $S_{2} \leftarrow S_{0}$ absorption band of phenanthrene. Holes are observed in the 0,0 transition (right-hand side) and the first vibronic band $\mathbf{4 0 0}$ $\mathrm{cm}^{-1}$ above the origin (left-hand side). In the upper part the laser is tuned to the center of the 0,0 transition, in the lower part the laser is tuned to the center of the first vibronic band. All spectra are scaled to the same height to emphasize the strong similarity of the hole shapes. 
are non-resonant holes. All holes are scaled to the same height to emphasize the striking similarity of all four hole shapes. Both resonant holes have the same width and we must conclude that the vibronic state $S_{2.1}$ decays not faster than the vibrationless state $S_{2,0}$. The width of a non-resonant hole is the average of the widths of the corresponding two resonant holes plus a contribution resulting from imperfect correlation between the inhomogeneous distributions of the burned and the probed transitions. Since all hole widths are equal we conclude that correlation between the inhomogeneous distributions of these two vibronic states is very good.

In a supersonic jet the homogeneous linewidth of the $S_{2,0} \leftarrow S_{0,0}$ transition of phenanthrene is 11 $\mathrm{cm}^{-1}$, corresponding to a lifetime of $0.5 \mathrm{ps}$ [22]. Our hole-burning measurement yields a homogeneous width of $67 \mathrm{~cm}^{-1}$, corresponding to a lifetime of $80 \mathrm{fs}$, for the same transition in an organic glass. This shortening of the lifetime by a factor of 6 is accompanied by a reduction of the energy gap between the origins of $S_{1}$ and $S_{2}$ from $6051 \mathrm{~cm}^{-1}$ in the isolated molecule to $5000 \mathrm{~cm}^{-1}$ in the glass. Yet another situation is found with phenanthrene in a durene host crystal [27]. Here the $S_{2,0} \leftarrow S_{0,0}$ absorption band is $200 \mathrm{~cm}^{-1}$ broad and displays sharp substructure. In this case the energy gap between $S_{1}$ and $S_{2}$ is $4700 \mathrm{~cm}^{-1}$. The crystal spectrum was explained with an intermediatelevel-structure (ILS) model [28]: The $S_{2}$ state couples strongly to a few vibrational levels of $S_{1}$. The resulting mixed states couple in a statistical way with the quasicontinuum of states, thus yielding a dense spectrum of lorentzian lines. The superposition of these lines leads to the broad absorption band with some fine structure superimposed. Well-resolved ILS spectra have been observed in supersonic jets for the $S_{2}$ states of naphthalene [29] and pyrene [30]. The energy gap to the $S_{1}$ state in these molecules is 3900 and $4000 \mathrm{~cm}^{-1}$, respectively.

The situation found in the glass is, therefore, dramatically different from both the isolatedmolecule case and the mixed-crystal case. In view of the similar energy gap for the crystal and the glass one could argue that also in the organic glass the ILS model applies. In this case hole-burning should yield a hole shape consisting of a broad band with a narrow spike in the center. The large width would correspond to the envelope of the ILS spectrum, whereas the width of the narrow spike would correspond to the width of the individual lines within the ILS spectrum. The situation is analogous to the measurement of autocorrelation functions of laser pulses containing short-time substructure [31]. These autocorrelation traces show a broad component and a narrow line in the center. The width of this narrow "coherent spike" is a measure of the duration of the substructure pulses, whereas the broad component measures the pulse envelope. The relative amplitudes of the narrow and the broad components depend on the ratio of the width of the substructure pulses to their average spacing in the case of the autocorrelation function. In the case of holeburning of an ILS spectrum the relative amplitudes of the narrow and the broad components depend on the ratio of the width of the individual lines to their average spacing. Only if the width of the individual lines becomes larger than the spacing, i.e. if the lines coalesce, will the central narrow pike disappear.

We have not observed any narrow spike of this kind and conclude that, if an ILS structure were present, its average spacing would be smaller than $10 \mathrm{~cm}^{-1}$. In addition, its envelope would be three times narrower than in the mixed crystal. We must, therefore, conclude that in going from the mixed crystal to the organic glass more is happening than just a small increase of the energy gap from 4700 to $5000 \mathrm{~cm}^{-1}$.

To gain more insight into this problem we plan to study phenanthrene in other host systems. In particular argon matrices seem interesting since the gap increases to $5500 \mathrm{~cm}^{-1}$ and the interaction with the environment is much smaller than in organic glasses. On the other hand, hole-burning applied to the mixed crystal could measure the inhomogeneous contribution to the absorption band as well as the width of the individual lines within the ILS spectrum. 


\section{Acknowledgement}

We are very grateful to Drs. $H$. Hippler and $K$. Luther, and Professor J. Troe for the loan of the optical multichannel analyzer. We also thank Professors A. Weller and F.P. Schäfer for support of this work, and Professor D. Haarer for bringing to our attention ref. [25].

\section{References}

[1] M. Kasha, Discussions Faraday Soc. 9 (1950) 14.

[2] F. Hirayama, T.A. Gregory and S. Lipsky, J. Chem. Phys. 58 (1973) 4696.

[3] T.A. Gregory, F. Hirayama and S. Lipsky, J. Chem. Phys. 58 (1973) 4697.

[4] T.A. Gregory and S. Lipsky, J. Chem. Phys. 65 (1976) 296.

[5] M.R. Topp, H.-B. Lin and K.-J. Choi, Chem. Phys. 60 (1981) 47.

[6] B. Nickel, Helv. Chim. Acta 61 (1978) 198.

[7] R.L. Fork, B.I. Greene and C.V. Shank, Appl. Phys. Letters 38 (1981) 671.

[8] G.A. Mourou and T. Sizer II, Opt. Commun. 41 (1982) 47.

[9] J.C. Diels, J.J. Fontaine, I.C. McMichael, B. Wilhelmi, W. Dietel and D. Kuhlke, Soviet J. Quantum Electron. 13 (1983) 1562 ,

[10] J.E. Midwinter and J. Warner, J. Appl. Phys. 38 (1967) 519.

[11] L.A. Halliday and M.R. Top, Chem. Phys. Letters 46 (1977) 8.

[12] B. Kopainsky, W. Kaiser and F.P. Schăfer, Chem. Phys. Letters 56 (1978) 458.
[13] S.J. Strickler and R.A. Berg, J. Chem. Phys. 37 (1962) 814.

[14] J.B. Birks and D.J. Dyson, Proc. Roy. Soc. A 275 (1963) 135.

[15] W.R. Ware and B.A. Baldwin, J. Chem. Phys. 40 (1964) 1703.

[16] J.B. Birks, Photophysics of aromatic molecules (Wiley-Interscience, New York, 1970) pp. 100-102, table 4.1

[17] B. Nickel and G. Roden, Ber. Bunsenges. Physik. Chem. 81 (1977) 281.

[18] G.C. Orner and M.R. Topp, Chem. Phys. Letters 36 (1975) 295.

[19] H. B. Lin and M.R. Topp, Chem. Phys. Letters 47 (1977) 442.

[20] H.-B. Lin and M.R. Topp, Chem. Phys. Letters 48 (1977) 251.

[21] M.R. Topp and H.-B. Lin, Chem. Phys. Letters 67 (1979) 273.

[22] A. Amirav, M. Sonnenschein and J. Jortner, J. Phys. Chem. 88 (1984) 5593.

[23] J.L. Richards and S.A. Rice, Chem. Phys. Letters 9 (1971) 444; J. Chem. Phys. 54 (1971) 2014.

[24] J. Friedrich and D. Haarer, Angew. Chem. 96 (1984) 96; Angew. Chem. Intern. Ed. 23 (1984) 113.

[25] R.M. Shelby and R.M. MacFarlane, Chem. Phys. Letters 64 (1979) 545.

[26] M. Abramowitz and I.A. Stegun, eds., Handbook of mathematical functions (Dover, New York, 1970) p. 295.

[27] G. Fischer, Mol. Cryst. Liquid Cryst. 6 (1969) 105.

[28] G. Fischer, Chem. Phys. 4 (1974) 62.

[29] S.M. Beck, D.E. Powers, J.B. Hopkins and R.E. Smalley, J. Chem. Phys. 73 (1980) 2019.

[30] E.A. Mangle and M.R. Topp, J. Phys. Chem. 90 (1986) 802.

[31] H.A. Pike and M. Hercher, J. Appl. Phys 41 (1970) 4562. 